\title{
Weekdays and Weekends Variation of Step Count in 9 to 11 years old obese school children
}

Corresponding author:

Dr. Sharifah Wajihah Wafa, School of Nutrition and Dietetics, Universiti Sultan Zainal Abidin, Malaysia, Kuala Terengganu, Terengganu

Tel (Office): +609 6688518

Tel (Mobile): +6012 6911510

Fax : +6096275639

sharifahwajihah@unisza.edu.my

Co-authors:

Ms. Nur Nadzirah Aziz

School of Nutrition and Dietetics, Universiti Sultan Zainal Abidin, Malaysia, Kuala Terengganu, Terengganu

nurnadzirah@gmail.com

Ms. Marhasiyah Rahim

School of Rehabilitation Sciences, Universiti Sultan Zainal Abidin, Malaysia, Kuala Terengganu, Terengganu

marhasiyah@unisza.edu.my 


\section{Abstract}

A major health threat for children in the 21st century is physical inactivity in conjunction with the elevated prevalence of obesity. In Malaysia, low physical activity among children is a major concern. The objective of the study was to analyze the day to day variation of step count in a sample of obese children using the activPAL ${ }^{T M}$ monitor. This was a cross sectional study conducted in Kuala Terengganu, Terengganu. The sample compromised 41 boys and 24 girls between the ages of 911 years. Number of steps was objectively measured using an activPAL ${ }^{\mathrm{TM}}$ accelerometer over a period of 4-7 days and had their height and weight measured. Mean step count from this sample of obese children was $8861 \pm 3157$ steps. Steps per day were significantly higher for boys compared to girls $(p=0.034)$. Obese children took significantly more steps at weekdays than on weekends $(p=0.001)$. Steps/hour were significantly higher in weekday compared to weekend between 0600 to $0700(p<0.001), 0700$ to $0800(p<0.001), 0800$ to 0900 am $(p<0.001), 0900$ to $1000(p=0.032), 1000$ to $1100(p=0.046), 1100$ to $1200(p<0.001), 1300$ to 1400 $(p=0.002), 1400$ to $1500(p<0.001), 1800$ to $1900(p=0.026)$ and 2000 to 2100 $(p=0.019)$. Detailed daily patterns of physical activity are required to fully understand the differences across days that help future interventions to target those falling short particularly during weekend.

Keywords: Childhood, obesity, activPAL, steps, sedentary behavior 


\section{Introduction}

Physical activity is beneficial to health as it promotes normal growth, and maintains physical fitness as well as psychological well-being of children and adolescents [1]. It has become major attention to the public health practitioners for its role in childhood obesity. The prevalence of childhood obesity has been escalating in both children and adult in Malaysia. The latest National Health and Morbidity Survey (NHMS 2015) reported that $11.9 \%$ of Malaysian children aged below 18 years old were obese [2]. The percentage of this epidemic has been increased by $5.8 \%$ in just four year time as compared to the previous NHMS 2011 [3].The increased prevalence of obesity should not be taken lightly, as childhood obesity was known to be a predictor of obesity in adults. Hazreen et al., further reported high sedentary lifestyle among adolescents from rural area in Malaysia which may contribute to the occurrence of adiposity [4]. Numerous studies reported that physical activity was inversely correlated with obesity [5-8]. The knowledge regarding the relationship between physical activity and obesity was important in order to tackle the childhood obesity problem in Malaysia [9].

Walking was a form of physical activity that often used by the researchers throughout the world to investigate the level of physical activity using various accelerometers [10]. An accelerometer is able to assess the physical activity for one day or several days including weekdays and weekends more accurately than selfreport as it removes the tendency to over-reports [11]. One of them was an activPAL ${ }^{\mathrm{TM}}$ monitor. Although there was dearth information regarding physical activity among primary school children in Malaysia, none of them had used the device for measuring their step counts. Therefore, the primary purpose of this study was to analyze the weekday and weekend variations of step count in a sample of obese 
children, residing in an urban area in the East Coast of Malaysia using the activPAL ${ }^{\mathrm{TM}}$ monitor.

\section{Methodology}

\section{Participants}

Full details of participant's recruitment and study procedure have been reported elsewhere [12]. In brief, the present study is a cross-sectional study measuring the daily activity of lying down, sitting, standing and stepping of obese of obese children in Kuala Terengganu, Malaysia. Data were collected between 2014 and 2015 at the Universiti Sultan Zainal Abidin (UniSZA), Terengganu. For the purpose of this study, the children aged 9 to 11 years from randomly selected primary schools in Kuala Terengganu had to be obese with BMI z-score more than +2 SD (WHO 2007 Growth Reference). Ethical approval was obtained from the UniSZA Human Research Ethics Committee (UHREC) (UniSZA.N/1/628-1 (28)) and permission for data collection was granted by Ministry of Education (Malaysia). Parental consent and child assent were obtained prior to data collection.

\section{Anthropometric Measurement}

During data collection day, their height was measured to the nearest $0.1 \mathrm{~cm}$, while their weight was measured to the nearest $0.1 \mathrm{~kg}$ using portable stadiometer (Seca 213) and digital weight scale (Seca Robusta 813), respectively. Then, BMI and BMI z-score was calculated using the WHO AntroPlus software version 1.0.4. The BMI zscores were then placed into the categories of severe thinness, thinness, normal 
weight, overweight and obese with the cut-off point of $<-3 S D,<-2 S D, \geq-2 S D$ to $\leq$ $+1 \mathrm{SD},>+1 \mathrm{SD}$ and > +2SD respectively based on the WHO 2007 Growth Reference. Only children classified as obese (+2 SD) were included in this study.

\section{ActivPAL ${ }^{\mathrm{TM}}$ Monitor}

The children were instructed to wear an activPAL ${ }^{\mathrm{TM}}$ monitor for 7 consecutive days including during sleeping. They were only allowed to remove the device during shower or any water-related activity. The children were fitted with the activPAL accelerometer, attached to the thigh using a double-sided hydrogel adhesive pad (PALstickies, PAL Technologies Ltd., Glasgow, UK) and covered by an elastic tube bandage or thigh support to ensure that the device stayed in place. The device has been shown to be an accurate, reliable and valid tool to measure step counts in children [13].

\section{Data Processing}

Only children that provided with at least 4 valid days (including one weekend day) were included in the study. One valid day was considered when the children wore the device for at least 600 minutes during waking hours $(6.00$ am to $11.00 \mathrm{pm})$. If the children failed to wear the device for at least 600 minutes during waking hours, then the whole day will be removed from the analysis. The average step counts for every waking hour (6.00 am- $11.00 \mathrm{pm}$ ) of the valid day, for each participant was calculated manually from the raw data provided by the activPAL ${ }^{\mathrm{TM}}$ software. 


\section{Statistical Analysis}

All the variables were tested for normality and were normally distributed. All the data were presented in the table as mean (SD). A paired t-test was applied to compare the difference of two means. The analysis was considered significant at a p-value less than 0.05. All data were analysed using IBM SPSS Statistics Version 20.0.

\section{Results}

A total of 65 obese children provided activPAL data and so were entered into analyses. Of the 65 obese children, $63 \%$ were boys and $37 \%$ were girls had a mean BMI $26.8 \pm 3.2 \mathrm{~kg} / \mathrm{m}^{2}$ with mean age $10.1 \pm 0.8$ years old. No significance difference was observed in the characteristics of the children between boys and girls (Table 1). The present study shows that boys significantly made more steps than girls $(9420 \pm 3285$ steps; $p=0.044)$. A similar trend was seen when the results were compared within gender.

Table 1: Characteristics of the children $(n=65)$

\begin{tabular}{|c|c|c|c|}
\hline Characteristic & $\begin{array}{l}\text { Full sample } \\
(n=65)\end{array}$ & $\begin{array}{l}\text { Boys } \\
(n=39)\end{array}$ & $\begin{array}{l}\text { Girls } \\
(n=26)\end{array}$ \\
\hline Age (years) & $10.1(0.8)$ & $10.2(0.7)$ & $10.0(0.9)$ \\
\hline \multicolumn{4}{|c|}{ Anthropometric measurements } \\
\hline Height $(\mathrm{cm})$ & $140.8(7.3)$ & $140.8(7.5)$ & $140.9(7.0)$ \\
\hline Weight (kg) & $53.7(10.2)$ & $52.4(10.5)$ & $55.5(9.7)$ \\
\hline BMI $\left(\mathrm{kg} / \mathrm{m}^{2}\right)$ & $26.8(3.2)$ & $26.2(3.3)$ & $27.7(2.7)$ \\
\hline BMI z-score & $2.9(0.6)$ & $3.0(0.7)$ & $2.9(0.4)$ \\
\hline
\end{tabular}

Data were presented as mean (SD) 
Between the hours of 0600 and 2300, the proportion of daily steps were significantly higher during weekdays compared to weekends (9189 vs 7797 steps; $p<0.01)$ (Table 2). A significant difference also existed when the results were compared within gender, with boys accumulating more steps during weekend compared to girls $(p<0.05)$ but no significant difference was observed during weekdays.

Table 2: Number of steps between weekday and weekend day and within gender $(n=65)$

\begin{tabular}{|c|c|c|c|c|c|c|}
\hline & \multicolumn{3}{|c|}{$\begin{array}{l}\text { Weekday } \\
\text { Mean (SD) }\end{array}$} & \multicolumn{3}{|c|}{$\begin{array}{l}\text { Weekend } \\
\text { Mean (SD) }\end{array}$} \\
\hline & $\begin{array}{c}\text { All } \\
(n=65)\end{array}$ & $\begin{array}{l}\text { Boys } \\
(n=41)\end{array}$ & $\begin{array}{c}\text { Girls } \\
(n=24)\end{array}$ & All & $\begin{array}{l}\text { Boys } \\
(n=41)\end{array}$ & $\begin{array}{c}\text { Girls } \\
(n=24)\end{array}$ \\
\hline $\begin{array}{c}\text { Number of } \\
\text { steps }\end{array}$ & $\begin{array}{c}9189 \\
(3289)\end{array}$ & $\begin{array}{c}9864 \\
(3446)\end{array}$ & $\begin{array}{c}8178 \\
(2804)\end{array}$ & $\begin{array}{c}7797 \\
(4116) *\end{array}$ & $\begin{array}{c}8337 \\
(4630) \dagger\end{array}$ & $\begin{array}{c}6988 \\
(3107) \dagger\end{array}$ \\
\hline
\end{tabular}

Mean values were significantly different between weekend and weekdays at ${ }^{*} p<$ 0.01 ; Mean values were significantly different between weekday and weekend within gender at $\dagger p<0.05$

Figure 1 shows there were significantly more number of steps accumulated during weekday compared to weekend between the hours of 0600 to $0700(p<0.001)$, 0700 to 0800,0800 to $0900(p<0.001), 0900$ to $1000(p=0.032), 1000$ to 1100 $(p=0.046), 1100$ to $1200(p<0.001), 1300$ to $1400(p=0.002), 1400$ to $1500 \mathrm{pm}$ $(p<0.001), 1800$ to $1900 \mathrm{pm}(p=0.026)$ and 2000 to $2100(p=0.019)$.

The children accumulated number of steps on weekday mostly around 10.00 am to 11.00 am with an average of 986 steps and around $1.00 \mathrm{pm}$ to $2.00 \mathrm{pm}$ with an average of 984 steps (Figure 2). However, during a weekend day, obese children 
were active mostly from $6.00 \mathrm{pm}$ to $7.00 \mathrm{pm}$ with an average of 584 steps and from 10.00 am to 11.00 am with an average of 562 steps (Figure 3).

Figure 2 showed that the respondents can achieve as high as 2517 steps for the time between 10.00 am to 11.00 am during weekday and 1995 steps for the time between $1.00 \mathrm{pm}$ to $2.00 \mathrm{pm}$. However, apart from that, the figure also showed that the respondents were also active at the time between 7.00 am to 8.00 am and the time between $6.00 \mathrm{pm}$ to $7.00 \mathrm{pm}$ which represented the time before going to school and the time to play, respectively. During the weekend day, most of the respondents were active around $6.00 \mathrm{pm}$ to $7.00 \mathrm{pm}$, with one of them can achieve a number of steps as high as 5026 steps (Figure 3).

\section{Discussion}

To the best of our knowledge, this is the only study of obese children physical activity assessed by activPAL accelerometer conducted in a South East Asian country. This Malaysian sample of 9 to 11 years old obese children took approximately 8782 total steps per day. They were reported to have low percentage of meeting the recommended daily step counts cut off points $(10.3 \%$ boys, $15.4 \%$ female)[14]. The averages detailed in the current study are most consistent with those reported in a large nationally representative sample of Malaysian children aged 7 to 12 years [9]. However, this sample of obese children was clearly more inactive when compared with other European studies, such as those from Ireland [15] and Greece [16] that have reported approximately 10000 to 13000 daily steps and was also less active than Australian obese children [17]. 


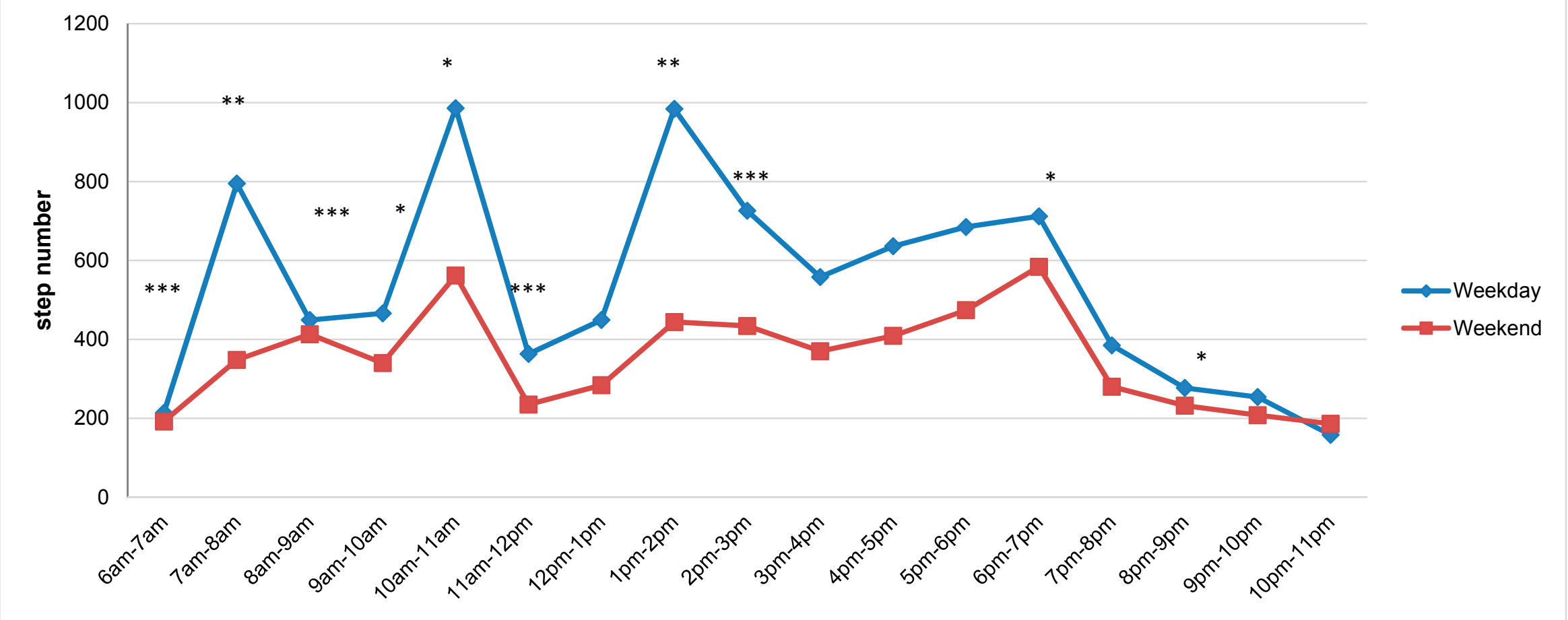

Mean values were significantly different between weekend and weekdays at ${ }^{*} p<0.05 ;{ }^{* *} p<0.01 ;{ }^{* * *} p<0.001$

Figure 1: Comparison number of steps between weekday and weekend day for every hour between $6.00 \mathrm{am}$ to $11.00 \mathrm{pm}$. 
3000

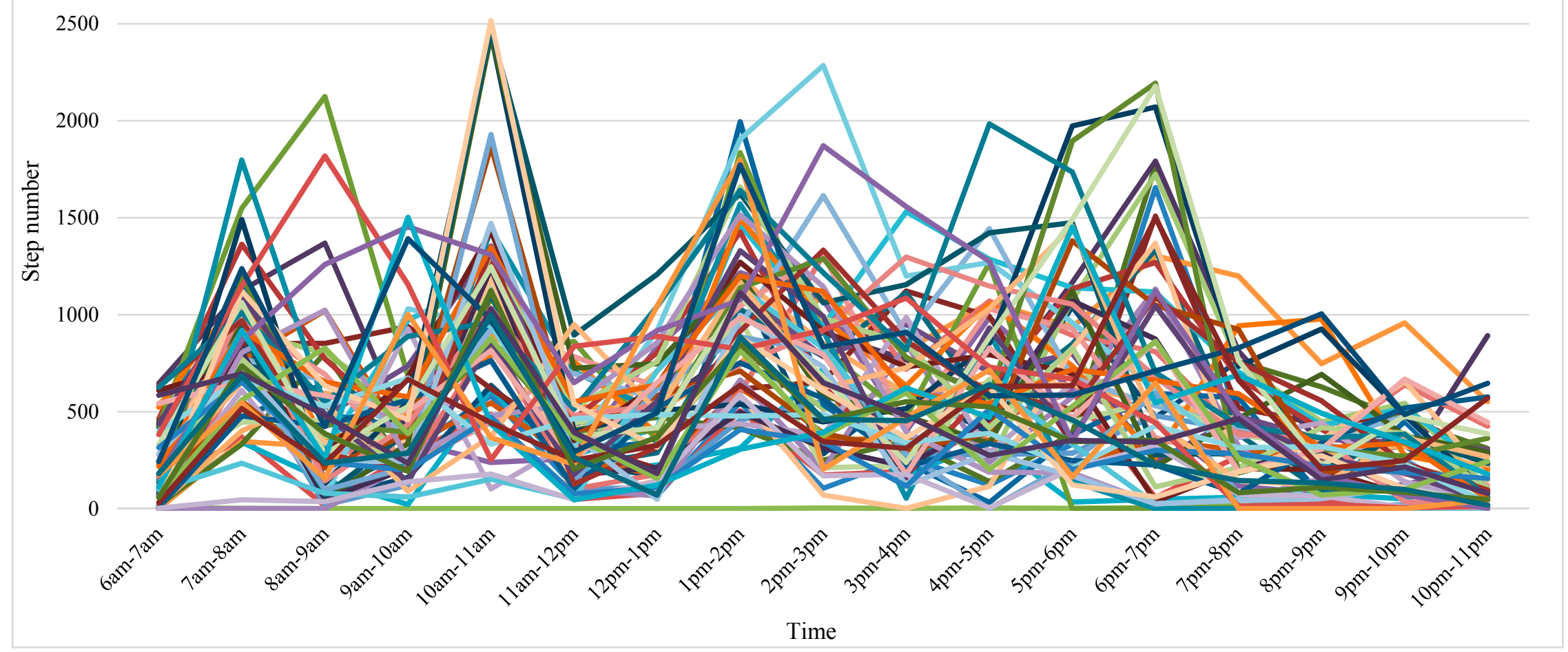

Figure 2: Inter-individual variation for hourly weekday steps between 6.00 am to $11.00 \mathrm{pm}$. Each line represents one participant 


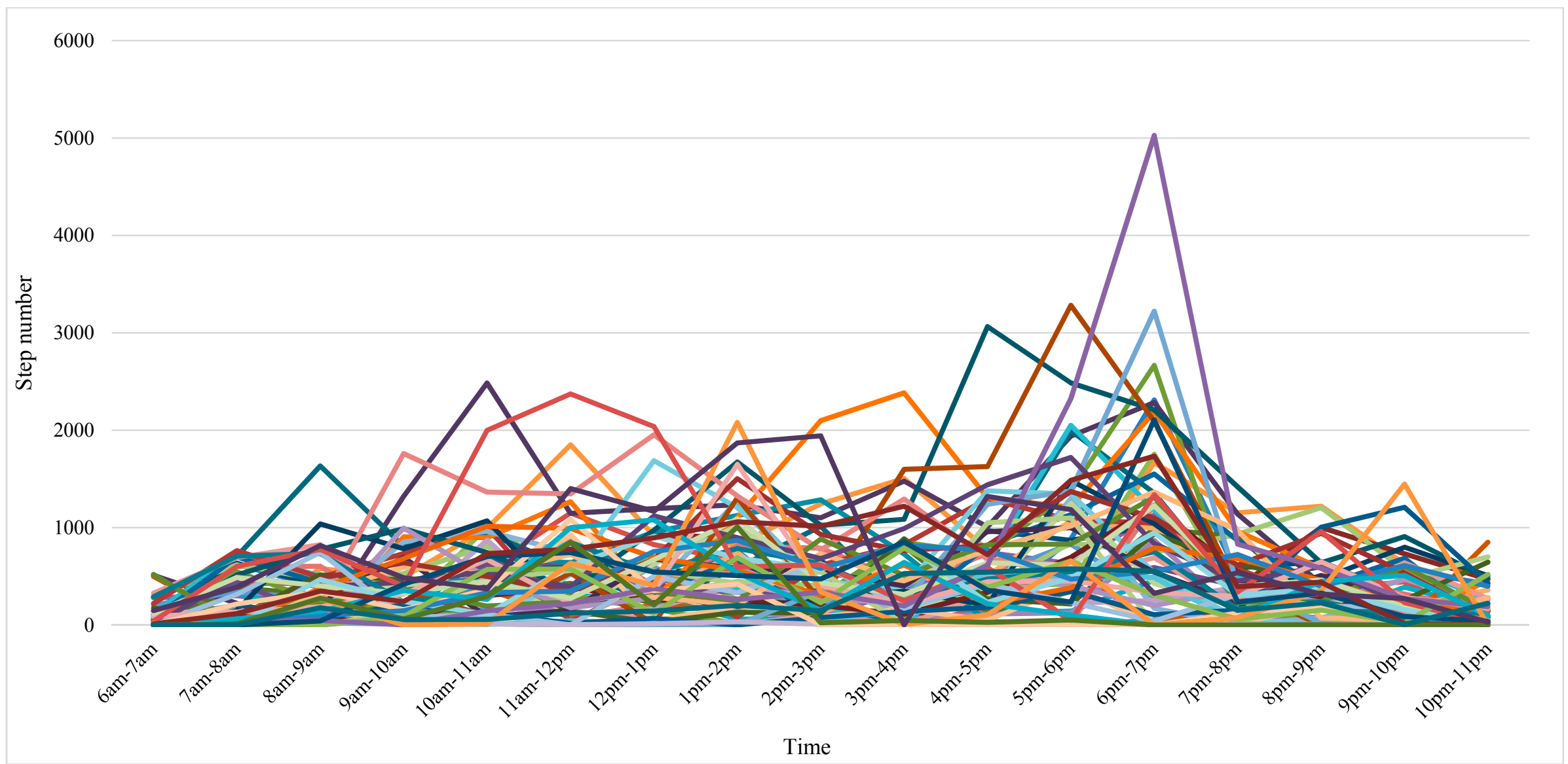

Figure 3: Inter-individual variation for hourly weekend steps between 6.00 am to $11.00 \mathrm{pm}$. Each line represents one participant. 
Obese children in the current study were also found to be significantly more active on weekdays than weekends on which again in contrast to the findings among Irish children [15]. This disparity in findings may be due to the fact that children in Malaysia have more opportunities to be physically active within the school environment and participation in after school curriculum programs provides them with more opportunities to be physically active, whereas, this may not be the case in other countries. Furthermore, physical activity outcomes were consistent with previous findings that the boys in the sample took more steps per day compared to girls [9,15-17]. Boys had assessed and more engaged in neighborhood physical activity while girls participated in more passive activities like socializing, instead involved directly in physical activity [18]. Moreover, boys were allowed to play more independently than girls, which explained why boys had more step counts than girls [18]. Although more steps were observed among boys (9420 steps/day for boys and 7826 steps/day for girls) this study suggests that neither, boys nor girls, meet the average daily steps count recommendations for elementary school children $(13,000$ steps for boys; 11,000 steps for girls) [14]

To examine sedentary time more accurately, few studies further split the day into different categories including before school, during school and after school $[19,20]$. The present study calculated number of steps hour by hour starting from 6.00 am until $11.00 \mathrm{pm}$ to further investigate the daily activities of these children. Children were also more active from 0700 to 0800,1000 to 1100 and $1300 \mathrm{pm}$ to $1400 \mathrm{pm}$ as those were the times that children having a break or recess during school hour and time they back from school or class ended. Furthermore, majority of the children were active at the time between $6.00 \mathrm{pm}$ to $7.00 \mathrm{pm}$ for both during weekdays and 
weekends which indicates that there was a peak hour they mostly play and move around with their peers.

The strength of this study was the fact that the activPAL ${ }^{T M}$ monitor was the most accurate device for assessing steps for all walking speeds as it has a high degree of accuracy and reliability [21]. The device was different than pedometers as it was not sensitive to small movements [13]. This means that activPAL ${ }^{T M}$ will not misinterpret fidgeting during watching television or sitting as steps, as long as their thigh did not move. Several limitations also should be noted regarding this study. First, the small age range of this study (9 to 11 years) and relatively small sample size. Also since the samples of participants were all drawn from schools in Kuala Terengganu, caution should be taken when generalizing these results to the 9-11 year old Malaysian population.

\section{Conclusion}

Through the detailed analysis of daily stepping this study has confirmed the obese children were more active on weekdays than weekends. Late evening (0600pm to 0700pm) was identified as the major contributor to the higher stepping observed during weekends. Additionally, the analysis revealed significant sex differences at overall steps/day in which boys having higher steps than girls for both weekdays and weekends. These findings are alarming due to very low activPAL determined physical activity levels of children's as proposed in the literature. Findings support the need for specific interventions targeting children's physical activity in an effort to improve these figures. 


\section{Acknowledgements}

The authors would like to thank the school, UniSZA's staff as well as undergraduate students for helping in recruitments and data collection process. We also would like to thank the children and their parents for participating in this study.

\section{Author Contributions}

NNA and MR were responsible for subject recruitment, screening, consenting of each family into the study and carried out every aspect of data collection. SWW and NNA participated in the study design, performed the statistical analysis and helped to draft the manuscript. All authors read and approved the final manuscript."

\section{Conflicts of interest}

The authors report no conflict of interest. The authors alone are responsible for the content and writing of the paper. 


\section{References}

1. Hills A.P., King N.A., Armstrong T.P. The contribution of physical activity and sedentary behaviours to the growth and development of children and adolescents: implications for overweight and obesity. Sports Med. New Zealand; 2007;37(6):533-45. DOI: 10.2165/00007256-200737060-00006.

2. Institute for Public Health. National Health and Morbidity Survey 2015 (NHMS 2015). Vol. II: Non-Communicable Diseases, Risk Factors \& Other Health Problems. 2015.

3. Institute for Public Health. National Health and Morbidity Survey 2011 (NHMS 2011). Vol. II: Non-Communicable Diseases. 2011.

4. Majid H.A., Amiri M., Mohd Azmi N., Su T.T., Jalaludin M.Y., Al-Sadat N. Physical activity, body composition and lipids changes in adolescents: analysis from the MyHeART Study. Sci Rep; 2016;6:30544. DOI: 10.1038/srep30544.

5. Remmers T., Sleddens E.F.C., Gubbels J.S., de Vries S.I., Mommers M., Penders J., et al. Relationship between physical activity and the development of body mass index in children. Med Sci Sports Exerc.; 2014 Jan;46(1):17784. DOI:10.1249/MSS.0b013e3182a36709.

6. Plonka M., Toton-Morys A., Adamski P., Suder A., Bielanski W., Dobrzanska M.J., et al. Association of the physical activity with leptin blood serum level, body mass indices and obesity in schoolgirls. J Physiol Pharmacol.; 2011 Dec;62(6):647-56.

7. Rauner A., Mess F., Woll A. The relationship between physical activity, physical fitness and overweight in adolescents: a systematic review of studies published 
in or after 2000. BMC Pediatr.; 2013;13:19. DOI:10.1186/1471-2431-13-19.

8. Kiranmala N., Das M.K., Arora N.K. Determinants of childhood obesity: need for a trans-sectoral convergent approach. Indian J Pediatr.; 2013 Mar;80 Suppl 1:S38-47. DOI:10.1007/s12098-013-0985-z

9. Lee S., Wong J., Shanita S., Ismail M., Deurenberg P., Poh B. Daily Physical Activity and Screen Time, but Not Other Sedentary Activities, Are Associated with Measures of Obesity during Childhood. Int J Environ Res Public Health. 2014;12(1):146-61. DOI:10.3390/ijerph120100146

10. Ryan C.G., Grant P.M., Tigbe W.W., Granat M.H. The validity and reliability of a novel activity monitor as a measure of walking. Br J Sports Med.; 2006 Sep;40(9):779-84.DOI: 10.1136/bjsm.2006.027276

11. Treuth M.S., Catellier D.J., Schmitz K.H., Pate R.R., Elder J.P., McMurray R.G., et al. Weekend and weekday patterns of physical activity in overweight and normal-weight adolescent girls. Obesity; 2007 Jul;15(7):1782-8. DOI: $10.1038 /$ oby.2007.212

12. Wafa S.W., Aziz N.N., Shahril M.R., Halib H., Rahim M., Janssen X. Measuring the Daily Activity of Lying Down, Sitting, Standing and Stepping of Obese Children Using the ActivPAL TM Activity Monitor. J Trop Pediatr. 2017; 63(2):98103. DOI: 10.1093/tropej/fmw052.

13. Aminian S., Hinckson E.A. Examining the validity of the ActivPAL monitor in measuring posture and ambulatory movement in children. IntJ Behav Nutr Phys Act. 2012;9:119. DOI: 10.1186/1479-5868-9-119

14. Tudor-Locke C., Craig C.L, Beets M.W., Belton S., Cardon G.M., Duncan S., et 
al. How many steps/day are enough for children and adolescents? Int J Behav Nutr Phys Act; 2011;8(1):78. DOI: 10.1186/1479-5868-8-78.

15. Belton S., Brady P., Meegan S., Woods C. Pedometer step count and BMI of Irish primary school children aged 6-9 years. Prev Med; 2010;50(4):189-92. DOI: 10.1016/j.ypmed.2010.01.009

16. Michalopoulou M., Gourgoulis V., Kourtessis T., Kambas A., Dimitrou M., Gretziou H. Step counts and body mass index among 9-14 years old greek schoolchildren. J Sport Sci Med. 2011;10(1):215-21.

17. Hands B., Parker H. Pedometer-determined physical activity, BMI, and waist girth in 7- to 16-year-old children and adolescents. J Phys Act Health. 2008;5 Suppl 1:S153-65.

18. Mitchell C.A., Clark A.F., Gilliland J.A. Built environment influences of children's physical activity: Examining differences by neighbourhood size and sex. Int $J$ Environ Res Public Health. 2016;13(1). DOI: 10.3390/ijerph13010130.

19. Dowd K.P., Harrington D.M., Bourke A.K., Nelson J., Donnelly A.E. The measurement of sedentary patterns and behaviors using the activPAL ${ }^{\mathrm{TM}}$ Professional physical activity monitor. Physiol Meas. 2012;33(11):1887-99. DOI: $10.1088 / 0967-3334 / 33 / 11 / 188$

20. Harrington D.M., Dowd K.P., Bourke A.K., Donnelly A.E. Cross-Sectional analysis of levels and patterns of objectively measured sedentary time in adolescent females. Int J Behav Nutr Phys Act; 2011;8(1):120. DOI:

10.1186/1479-5868-8-120। 
21. Oliver M. Counting Steps in Research: A Comparison of Accelerometry and Pedometry. Open J Prev Med. 2011;01(01):1-7. DOI:

10.4236/ojpm.2011.11001 